Björn Hayer, Klarissa Schröder (Hg.)

\title{
Tierethik transdisziplinär
}

Literatur - Kultur - Didaktik

[transcript] 


\section{Bibliografische Information der Deutschen Nationalbibliothek}

Die Deutsche Nationalbibliothek verzeichnet diese Publikation in der Deutschen Nationalbibliografie; detaillierte bibliografische Daten sind im Internet über http://dnb.d-nb.de abrufbar.

\section{(c) 2018 transcript Verlag, Bielefeld}

Alle Rechte vorbehalten. Die Verwertung der Texte und Bilder ist ohne Zustimmung des Verlages urheberrechtswidrig und strafbar. Das gilt auch für Vervielfältigungen, Übersetzungen, Mikroverfilmungen und für die Verarbeitung mit elektronischen Systemen.

Umschlaggestaltung: Kordula Röckenhaus, Bielefeld Umschlagabbildung: »Du sollst nicht töten«, Karl Wilhelm Diefenbach 1902, Städel Museum Frankfurt a.M.

Druck: Majuskel Medienproduktion GmbH, Wetzlar

Print-ISBN 978-3-8376-4259-9

PDF-ISBN 978-3-8394-4259-3

https://doi.org/10.14361/9783839442593

Gedruckt auf alterungsbeständigem Papier mit chlorfrei gebleichtem Zellstoff. Besuchen Sie uns im Internet: https://www.transcript-verlag.de Bitte fordern Sie unser Gesamtverzeichnis und andere Broschüren an unter: info@transcript-verlag.de 
This text was published in: Hayer, Björn; Schröder, Klarissa (eds.), Tierethik transdisziplinär : Literatur - Kultur - Didaktik, transcript Bielefeld, 2019;

https://doi.org/10.14361/9783839442593-023.

It is posted here by permission of transcript Verlag for personal use only, not for redistribution.

\title{
Tierethische und literaturdidaktische Potenziale in Paul Maars Wiedersehen mit Herrn Bello
}

\author{
Andreas Wicke
}

\section{EINLEITUNG}

Kein Schulfach ist so vielfältig wie der Deutschunterricht, der jedes Thema eines literarischen Textes auch zum Unterrichtsthema machen kann. Zwar ist Tierethik kein primärer Gegenstand des Fachs Deutsch, allerdings verbindet Literatur die Ebenen von histoire und discours, neben dem >Was` steht also immer auch das >Wie` der Darstellung. Im Idealfall bieten literarische Texte über Tiere respektive Tiere in Texten so die Möglichkeit, themen- und problemorientierten Unterricht sowie literarisches Lernen miteinander zu verbinden und damit die kulturwissenschaftlichen Ansätze der Human- oder Literary Animal Studies auch in den Deutschunterricht zu transportieren. Wie das in den Jahrgangsstufen 4 bis 6 aussehen kann, soll exemplarisch an Paul Maars Roman Wiedersehen mit Herrn Bello (2008), dem letzten Band der Herr Bello-Trilogie, gezeigt werden.

»In der Stadt kam uns ein ganzes Rudel von Kleinen entgegen. Ihr Herrchen hatte sie Gassi geführt «, ${ }^{1}$ erzählt der Hund Bello gleich zu Beginn des Romans und meint damit, dass er eine Schulklasse und ihren Lehrer gesehen hat, die einen Ausflug machen. Bereits hier ist man mitten in einer Handlung, die mit Perspektivwechseln und Speziesgrenzen, aber auch mit dem Verhältnis von Sprache und Wirklichkeit spielt. In den Romanen um den Hund Bello, der sich in einen Menschen verwandelt, stehen die menschliche und die tierliche Sicht einander direkt gegenüber. Eine >richtige` Perspektive existiert nicht, durch die ständigen Verwandlungen sitzt der Hund Bello bzw. der Mensch Herr Bello ohnehin zwischen allen Stühlen und Kategorien.

1 Maar, Paul: Wiedersehen mit Herrn Bello, Hamburg: Oetinger 2008, S. 8. 
Perspektivwechsel gehören zum narrativen Basisrepertoire Paul Maars, immer wieder erzählt er Geschichten aus unterschiedlichen und unerwarteten Sichtweisen. Zwar kann man ihn nicht als Verfasser explizit tierethischer Texte bezeichnen, allerdings durchzieht die Sensibilisierung für verschiedene Formen des Zusammenlebens von Menschen und Tieren sein Gesamtwerk und führt zu einem impliziten Appell für die Rechte von Tieren. Bereits in seiner ersten kinderliterarischen Publikation, Der tätowierte Hund von 1968, erzählt Maar bzw. der Löwe das grimmsche Märchen Hänsel und Gretel aus der Sicht der Hexe, sodass die Kategorien >gut >böse< verschwimmen. In der anschließenden Rahmenhandlung heißt es: '»Wenn das so ist‘, überlegte der Hund, >dann möchte ich gerne einmal Rotkäppchen von einem Wolf erzählt bekommen « ${ }^{2}{ }^{2}$ Das ist zunächst ein raffiniert-witziger Einfall für ein Kinderbuch, darüber hinaus aber auch die Idee, den Wolf von dem Stigma >böses Tier $\mathrm{zu}$ befreien und ihm außerdem eine Stimme zu verleihen. ${ }^{3}$

\section{PERSPEKTIVWECHSEL}

Auch in seiner Herr Bello-Trilogie spielt Paul Maar mit Perspektiven: Bello bzw. Herr Bello, wie er sich als Mensch nennt, ist ein Hybrid; im ersten Band taucht er zunächst als >normaler Hund auf, wird dann aber durch ein Elixier in einen Menschen verwandelt. Er nimmt also sowohl die menschliche als auch die tierliche Perspektive ein. Weil die Wirkung immer wieder nachlässt und aufgefrischt werden muss, sind die Grenzen fließend und es gibt diverse Zwischenstadien, sodass die Klassifizierung als »Hundemensch «, ${ }^{4}$ die in Wiedersehen mit Herrn Bello verwendet wird, durchaus zutreffend ist. ${ }^{5}$

Allerdings bemerkt Bello schnell, dass ein hybrides Dasein mit Problemen verbunden ist: »Die meisten Hundekollegen sind freundlich und wollen mit mir rennen«, sagt er. »Aber irgendwie fühle ich, dass ich nicht mehr dazugehöre. Das

2 Maar, Paul: Der tätowierte Hund, Reinbek: Rowohlt ${ }^{3} 2007$, S. 34.

3 Zum Bild des Wolfs in der Literatur vgl. Borgards, Roland: »Tiere und Literatur«, in: Ders. (Hg.), Tiere. Kulturwissenschaftliches Handbuch, Stuttgart: Metzler 2016, S. 225 244, hier S. 225f.

4 P. Maar: Wiedersehen mit Herrn Bello, S. 12 u. 125.

$5 \mathrm{Zu}$ einer differenzierteren Darstellung, die die kulturwissenschaftliche Sicht der HumanAnimal Studies mit einer intertextuellen Analyse der Herr Bello-Trilogie verbindet, vgl. Wicke, Andreas: »)Mönschsein ist gut`, sagte Herr Bello. >Aber Hundsein ist auch gut. Mensch-Tier-Perspektiven in Paul Maars Herr Bello-Trilogie«, in: Ders./Nikola Roßbach (Hg.), Paul Maar. Studien zum kinder- und jugendliterarischen Werk, Würzburg: Königshausen \& Neumann 2017, S. 121-138. 
Dumme ist, dass ich auch nicht zu den Menschen gehöre «. ${ }^{6}$ Während Bello zwischendurch ein Mensch war, hat er im dritten Teil eine menschliche Sprech- und Denkfähigkeit, ist jedoch wieder in Hundegestalt. „Von außen bin ich der Hund Bello. Innerlich der Herr Bello, der ich mal war «, ${ }^{7}$ resümiert er.

Wenn es den Human-Animal Studies darum geht, Tiere als ebenbürtigen Teil der Gesellschaft zu akzeptieren, dann müssen, so die grundlegende Forderung des Arbeitskreises Chimaira, »nichtmenschliche Tiere aus den Natur/Kultur-, Aktiv/Passiv- und Mensch/Tier-Dualismen herausgelöst werden «. ${ }^{8}$ Nun ist es ein Privileg der Literatur, Wesen zu schaffen, die gleichsam automatisch außerhalb der genannten Dualismen stehen und dazu führen, die Speziesgrenzen, wie Jacques Derrida es in Das Tier, das ich also bin beschreibt, »zu verkomplizieren, zu verdicken, zu entlinearisieren, zu krümmen, zu teilen «. ${ }^{9}$

Einem in menschlicher Sprache redenden Hund kommt darüber hinaus eine besondere Handlungsmacht zu, weil die Grenze zwischen sprechendem Menschen und stummem Tier hier aufgehoben ist, Bello wird dadurch zum tierlichen Akteur. In den drei Romanen experimentiert Paul Maar außerdem mit multiperspektivischen Erzählmustern: Während es im ersten Band neben Max als Ich-Erzähler noch eine auktoriale Erzählinstanz gibt, bekommt im dritten Band zusätzlich der Hund Bello eine Stimme, sodass die Gleichberechtigung auch erzähltheoretisch realisiert wird, vor allem, wenn einzelne Ereignisse aus verschiedenen Sichtweisen erzählt werden. Das hat nicht nur eine komische Wirkung, vielmehr kann dem narratologischen Perspektivwechsel ein ideologischer folgen: Sprechende Hunde haben in der Literatur spätestens seit E.T.A. Hoffmanns Nachricht von den neuesten Schicksalen des Hundes Berganza immer wieder zu einer Kritik an der alleinigen Würde des Menschen geführt und das herrschende Konzept von Menschsein destabilisiert:

»Ich bin zwar ein Hund, aber Euer Vorzug aufrecht zu gehen, Hosen zu tragen und beständig zu schwatzen, wie es Euch gefällt, ist nicht so viel wert, als im langen Schweigen den treuen

6 P. Maar: Wiedersehen mit Herrn Bello, S. 8.

7 Ebd., S. 12.

8 Chimaira Arbeitskreis: »Eine Einführung in Gesellschaftliche Mensch-Tier-Verhältnisse und Human-Animal Studies«, in: Chimaira - Arbeitskreis für Human-Animal Studies (Hg.), Human-Animal Studies. Über die gesellschaftliche Natur von Mensch-TierVerhältnissen, Bielefeld: transcript 2011, S. 7-42, hier S. 19.

9 Derrida, Jacques: Das Tier, das ich also bin, hg. v. Peter Engelmann, übers. v. Markus Sedlaczek, Wien: Passagen-Verlag 2010, S. 55. 
Sinn zu bewahren, der die Natur in ihrer heiligsten Tiefe ergreift und aus dem die wahre Poesie emporkeimt. ${ }^{10}$

In ihrer Analyse nicht-menschlicher Erzählinstanzen schreiben Lars Bernaerts et al. tierlichen Erzählern eine doppelte Funktion zu:

»Non-human narrators prompt readers to project human experience onto creatures and objects that are not conventionally expected to have that kind of mental perspective (in other words, readers >empathize (and >naturalize ); at the same time, readers have to acknowledge the otherness of non-human narrators, who may question (defamiliarize) some of readers' assumptions and expectations about human life and consciousness. $\ll^{11}$

Das hier diagnostizierte Spannungsfeld von Empathie und Verfremdung wird in Maars Herr Bello-Texten potenziert, da menschliche und nicht-menschliche Erzählinstanzen multiperspektivisch verbunden werden und Bello die Grenze zwischen Mensch und Tier immer wieder metamorphotisch torpediert.

\section{TIER-MENSCH-BEZIEHUNGEN}

In den Herr Bello-Romanen werden drei Formen des Zusammenlebens zwischen Menschen und Tieren präsentiert: Zunächst wird, vor allem zu Beginn des ersten Bandes, eine traditionelle Heimtierhaltung gezeigt, die einerseits durchaus liebevoll ist, andererseits aber von einer klaren Hierarchie ausgeht: »Dann hast du also ab heute einen Hund «, ${ }^{12}$ sagt Max' Vater und betont damit das anthropozentrische Besitzverhältnis, außerdem werden jene Herrschaftsinsignien angeschafft, die das Machtverhältnis unterstreichen: Vater und Sohn kaufen »ein Halsband, eine Leine [...] und das Buch >Wie erziehe ich meinen Hund? « ${ }^{13}$ Flankiert wird dieses Mensch-Tier-Verhältnis durch die weitgehend gleichberechtigte Freundschaft, die sich zwischen Max und Bello entwickelt, sowie im dritten Band durch die brutale Behandlung im Zirkus.

Das freundschaftliche Verhältnis zwischen Mensch und Tier wird immer wieder betont und es kommt zu kameradschaftlichen Aushandlungsprozessen, etwa, wenn

10 Hoffmann, E.T.A.: Fantasiestücke in Callot's Manier. Werke 1814, hg. v. Hartmut Steinecke, Frankfurt a.M.: Deutscher Klassiker Verlag 2006, S. $119 f$.

11 Bernaerts, Lars/Caracciolo, Marco et al.: »The Storied Lives of Non-Human Narrators«, in: Narrative 22 (2014) 1, S. 68-93, hier S. 69.

12 Maar, Paul: Herr Bello und das blaue Wunder, Hamburg: Oetinger 2005, S. 52.

13 Ebd., S. 63. 
Max eine Schulfreundin einladen will und Bello fragt, ob er etwas dagegen hat. $»$ Nein, nichts dagegen $«,{ }^{14}$ antwortet der. Selbst in Phasen der Meinungsverschiedenheit wird das Freundschaftsmodell als Maßstab herangezogen. »Freunde wünschen ihren Freunden nicht so was Fieses«, sagt Max, als Bello sich kritisch über jenes Mädchen äußert, in das Max verliebt ist. Wenn er jedoch in ein traditionelles Herrchen-Verhalten zurückfällt, echauffiert sich Bello entsprechend: »Bello, sitz! So kann man vielleicht mit einem Hund reden. Aber nicht mit einem Freund! Ich tat so, als wäre ich nicht gemeint. ${ }^{15}$ Stellenweise ähnelt die Freundschaft zwischen Max und Bello jenen »kameradschaftlichen Beziehungen «, die Donna Haraway beschreibt. »Tatsächlich finde ich«, sagt sie über die »companion species«, »dass diese Idee - sie ist weniger eine Kategorie als vielmehr Hinweis auf ein ständiges gemeinsam-Werden - ein viel ergiebigeres Geflecht ist, um darin zu leben. ${ }^{16}$

Ganz anders sieht die Behandlung des sprechenden Hundes im Zirkus Binotto aus. Hier wird das egalitäre bzw. kameradschaftliche Verhältnis zwischen Menschen und Tieren, zwischen Max und Bello durch ein hegemoniales und gewalttätiges kontrastiert. Bello wird vom Mitarbeiter eines Zirkus brutal entführt, um als sprechender Hund zur Publikumsattraktion dressiert zu werden. Da Bello sich weigert, mit dem Dompteur zu sprechen, wird er in einen zu kleinen Käfig gesperrt und soll mit Futterentzug zum Sprechen gezwungen werden. »Denk nur nicht, dass du etwas zu fressen bekommst«, sagt der Dompteur. »Du hungerst so lange, bis du den ersten Satz gesagt hast «. ${ }^{17}$

Der Zirkus als Institution geht allgemein von einem asymmetrischen Verhältnis aus, in dem das Tier als Objekt angesehen und zu Vergnügungszwecken gebraucht wird. Im konkreten Fall kommt allerdings eine physische Gewaltausübung hinzu. ${ }^{18}$ Bello selbst beschwert sich: »Es ist schlimm, wenn ein Hund nicht rennen kann, nur sitzen oder hin und her gehen $\ll .{ }^{19}$

14 P. Maar: Wiedersehen mit Herrn Bello, S. 17.

15 Ebd., S. 9.

16 Haraway, Donna: »Die Begegnung der Arten«, in: Roland Borgards/Esther Köhring et al. (Hg.), Texte zur Tiertheorie, Stuttgart: Reclam 2015, S. 290-325, hier S. 299.

17 P. Maar: Wiedersehen mit Herrn Bello, S 118.

18 Vgl. Buschka, Sonja/Gutjahr, Julia et al.: »Gewalt an Tieren«, in: Christian Gudehus/Michaela Christ (Hg.), Gewalt. Ein interdisziplinäres Handbuch, Stuttgart/Weimar 2013, S. 75-83. Vgl. auch Brando, Sabrina: »Zirkus«, in: Arianna Ferrari/Klaus Petrus (Hg.), Lexikon der Mensch-Tier-Beziehungen, Bielefeld: transcript 2015, S. 431-436 sowie Kompatscher, Gabriela/Spannring, Reingard et al.: Human-Animal Studies. Eine Einführung für Studierende und Lehrende, Münster/New York: Waxmann 2017, S. 93.

19 P. Maar: Wiedersehen mit Herrn Bello, S. 151. 
In $\S 1$ des deutschen Tierschutzgesetzes heißt es: »Niemand darf einem Tier ohne vernünftigen Grund Schmerzen, Leiden oder Schäden zufügen.« In § 2 wird konkretisiert: »Wer ein Tier hält, betreut oder zu betreuen hat, (1) muss das Tier seiner Art und seinen Bedürfnissen entsprechend angemessen ernähren, pflegen und verhaltensgerecht unterbringen« und »(2) darf die Möglichkeit des Tieres zu artgemäßer Bewegung nicht so einschränken, dass ihm Schmerzen oder vermeidbare Leiden oder Schäden zugefügt werden . $^{20}$

Daraus lassen sich im konkreten Fall ganz unterschiedliche Anklagepunkte formulieren, vor allem aber ist Bellos Unterbringung nicht artgerecht und er wird nicht ausreichend ernährt. »Ihr seht doch, wie Bello sich quält! «, ${ }^{21}$ sagt die Tochter des Zirkusdirektors bereits kurz nach seiner Ankunft. Als Orte der Gewalt gegen Tiere sind Schlachthäuser und Labore natürlich präsenter, aber auch der Zirkus stellt Tiere in einen funktionalen Kontext, wenn sie dort zu Unterhaltungszwecken dressiert werden.

\section{MENSCH - TIER - SPRACHE}

Mit den unterschiedlichen Mensch-Tier-Verhältnissen sind entsprechende sprachliche Konsequenzen verbunden. »Hunde sprechen hundlich, Mönschen sprechen mönschlich «, ${ }^{22}$ hatte Herr Bello im ersten Band verkündet, im dritten Band sagt Paloma: »Du musst dich nicht >entscholdigen<. Ich bin tierische Gerüche gewöhnt. Was glaubst du, wie unsere Ziegen stinken! « ${ }^{23}$ Die Adjektive >tierisch` und >hündisch bzw. >tierlich und >hundlich geben eine je andere Sichtweise wieder. Während Paloma offensichtlich nichts von den Sprachkonventionen der Human-Animal Studies weiß, ist Bello sprachlich sensibilisiert. Er reflektiert die pejorative Semantisierung von >hündisch` und stellt der >menschlichen` Sprache deswegen eine >hundliche` gegenüber. Arthur Schopenhauer weist darüber hinaus bereits im 19. Jahrhundert auf »die Eigenheit mancher Sprachen, namentlich der deutschen« hin,

»daß sie für das Essen, Trinken, Schwangerseyn, Gebären, Sterben und den Leichnam der Thiere ganz eigene Worte haben, um nicht die gebrauchen zu müssen, welche jene Akte beim

20 Tierschutzgesetz in der Fassung der Bekanntmachung vom 18. Mai 2006 (BGBl. I S. 1206, 1313), das zuletzt durch Artikel 141 des Gesetzes vom 29. März 2017 (BGB1. I S. 626) geändert worden ist.

21 P. Maar: Wiedersehen mit Herrn Bello, S. 117.

22 P. Maar: Herr Bello und das blaue Wunder, S. 73.

23 P. Maar: Wiedersehen mit Herrn Bello, S. 141. 
Menschen bezeichnen, und so unter der Diversität der Worte die vollkommene Identität der Sache zu verstecken. $\ll^{24}$

Auch in Maars Roman spielen solche Differenzen eine Rolle: »An deinen Pfoten wird das Fell ein bisschen grün«, sagt Max und Bello erwidert: »Nicht nur an meinen Füßen«. ${ }^{25}$ »Mit dem Mund«, so erklärt Bello, wie er es geschafft hat, eine Schublade aufzuziehen. »Du meinst: mit der Schnauze $«,{ }^{26}$ korrigiert Sternheim. Während Bello versucht, die sprachliche Grenze zu überwinden und die Distanz zwischen Menschen und Tieren aufzuheben, verwenden Max und sein Vater zwar die semantisch richtigen Begriffe, praktizieren damit allerdings eine Form des Othering, indem sie die tierliche Sphäre explizit von der menschlichen separieren. Dieser $»[\mathrm{~d}]$ istanzierende[] Sprachgebrauch ist zugleich speziesistisch, da Tiere aufgrund ihrer Nichtzugehörigkeit zur Spezies Mensch, lexikalisch anders bezeichnet und behandelt werden $\ll .{ }^{27}$

Auch wenn die tierethische Dimension in Paul Maars Texten nicht im Vordergrund steht, zeigt die exemplarische Analyse, dass er seine Geschichten hochbewusst komponiert und seine Erzählkonstruktionen konsequent durchdacht sind. Diese raffinierte Abstimmung von histoire und discours macht nicht nur eine besondere Qualität innerhalb der Kinderliteratur aus, Maars Werke erweisen sich auch als vielschichtige und anspruchsvolle Grundlage für den Deutschunterricht. Ein weiterer Vorteil ist, dass seine Texte ihre Ethik nicht gewaltsam diktieren, sondern verschiedene Positionen zur Diskussion stellen.

\section{METHODISCHE MÖGLICHKEITEN}

Aus diesen wenigen Überlegungen lassen sich ganz unterschiedliche Themenfelder für einen Deutschunterricht ableiten, der literarisches und tierethisches Lernen miteinander verknüpft. Vier Herangehensweisen - analytisch, textproduktiv, kreativ und szenisch - sollen hier exemplarisch skizziert werden:

24 Schopenhauer, Arthur: »Preisschrift über die Grundlage der Moral«, in: Ders.: Zürcher Ausgabe. Werke in zehn Bänden, Zürich: Diogenes 1977, Bd. VI, S. 143-315, hier S. 279.

25 P. Maar: Wiedersehen mit Herrn Bello, S. 14.

26 Ebd., S. 54.

27 Heuberger, Reinhard: »Sprachgebrauch: Das Mensch-Tier-Verhältnis aus linguistischer Sicht«, in: Kompatscher/Spannring et al., Human-Animal Studies (2017), S. 48-54, hier S. 50 . 
Bei einem erzähltheoretisch komplexen Text bieten sich natürlich analytische Zugänge an, die die multiperspektivische Erzählstruktur thematisieren; dabei geht es auch um die Frage, wie Maar traditionelle Mensch-Tier-Relationen erzähltheoretisch unterläuft und dekonstruiert. Wahrheit ist eben nicht monoperspektivisch gesetzt, sondern ergibt sich aus den unterschiedlichen Sichtweisen, die in den Herr Bello-Romanen raffiniert nebeneinander gestellt und gegeneinander montiert werden. Bei der Betrachtung der narratologischen Faktur wird deutlich, dass die menschliche und tierliche Perspektive sich oft widersprechen. Die jeweiligen Sichtweisen mit Blick auf die erzähltheoretische Realisierung zu untersuchen, ist ein lohnendes Thema im Deutschunterricht.

Im fantastischen Motiv des sprechenden Hundes liegt also ein emanzipatorisches Potenzial, weil die Sprechfähigkeit beider Spezies ein gleichberechtigtes Miteinander ermöglicht. Im realistischen Kinderbuch hingegen entstehen Missverständnisse oft, wenn Menschen sprechen und Hunde bellen. Als Beispiel kann etwa der Blick in Enid Blytons Kinderdetektivserie Fünf Freunde dienen - und Freund Nummer 5 ist bekanntlich Timmy, der Hund, der in der neuesten Übersetzung allerdings Tim heißt:

»Tim bellte aufgeregt, als sie in die Nähe der Höhle kamen. Er bellte unaufhörlich - laut und fordernd. `Es klingt ziemlich ärgerlich`, stellte Richard fest. `Armer Tim! Er hat sicher gedacht, wir hätten ihn vergessen.`Sie kamen an die Wagen, und Tim stürzte sich auf Georg, als habe er sie jahrelang nicht gesehen. Er stieg an ihr hoch, wollte ihr das Gesicht lecken, wedelte wie verrückt mit dem Schwanz und stieß hohe, sich überschlagende, klagende Töne aus. $\ll^{28}$

Erst allmählich merken die Kinderdetektive, dass der Hund Tim eine Spur gewittert hat, die er ihnen zeigen möchte. Mit einem sprechenden Hund würde die Szene gänzlich anders verlaufen, aber auch die Narration aus der Perspektive des Hundes würde die Geschichte wesentlich verändern. Durch solche vergleichenden Analysen sollen die Schülerinnen und Schüler einerseits ein Bewusstsein für erzähltheoretische Phänomene und Besonderheiten entwickeln, andererseits aber auch das anthropozentrische Machtgefälle zwischen Menschen und Tieren, zwischen menschlicher und tierlicher Sprache kritisch hinterfragen.

Mit der strukturellen Analyse lassen sich textproduktive Aufgaben ideal kombinieren. Indem die Schülerinnen und Schüler Passagen des Romans aus einer anderen Perspektive schreiben, erweitern sie nicht nur ihre Perspektivübernahmekompetenz, sondern müssen sich auch in die jeweilige tierliche oder menschliche Erzähl-

28 Blyton, Enid: Fünf Freunde beim Wanderzirkus, übers. v. Werner Lincke, München: cbj ${ }^{42} 1997$, S. $99 f$. 
instanz hineinversetzen. Eine anspruchsvollere Modellierung wäre, die Schülerinnen und Schüler den Fortgang der Geschichte skizzieren zu lassen, wenn nicht Bello, sondern Max den Saft getrunken und sich in einen Hund verwandelt hätte und Bello nun als Erzähler fungieren würde.

Aufgabensettings, die die Perspektive des Tieres ins Zentrum rücken und in denen die Kinder sich in die Rolle des Hundes versetzen, sensibilisieren die Schülerinnen und Schüler für die tierliche Perspektive. Es bleibt allerdings die Frage, ob ein von einem menschlichen Autor erfundenes sprechendes bzw. erzählendes Tier überhaupt ein Tier ist und ob hier die menschliche Perspektive nicht wiederum das tierliche Sprechen und Handeln dominiert. ${ }^{29}$

Komische Begebenheiten wie die eingangs zitierte Episode des Gassi gehenden Rudels ${ }^{30}$ können darüber hinaus als kreative Schreibimpulse genutzt werden, um eigene Geschichten über Menschen aus tierlicher Sicht zu schreiben. Dabei können die Schülerinnen und Schüler von jenen Beispielen ausgehen, in denen die Sprache zwischen Menschen und Tieren lexikalisch differenziert: >essen` oder >fressen<, >Pfote schreiben, die unter Menschen spielt, jedoch von einem Hund beobachtet und erzählt wird, besonders Essensszenen sind dafür geeignet. In der Besprechung der Ergebnisse wird es vor allem um Sprachreflexion gehen. Den Schülerinnen und Schülern soll bewusst werden, dass der Sprachgebrauch immer auch bestimmte - meistens anthropozentrische - Sichtweisen transportiert und dass Sprache, bekannter ist das Phänomen aus der Gender-Linguistik, in der Lage ist, konventionelle Hierarchien zu zementieren oder aufzubrechen. ${ }^{31}$

In Kirsten Boies Bilderbuch Josef Schaf will auch einen Menschen deutet sich der Perspektivwechsel bereits im Titel an und Klarissa Schröder hat entsprechende Aufgaben für die Primarstufe modelliert. ${ }^{32}$ Auch mit Blick auf Maars Roman ließe sich die Geschichte eines Hundes schreiben, der sich einen Hausmenschen wünscht, ihm eine Menschenhütte baut, ihn ausführt usw. Analog zu dem fiktiven Sachbuch Wie erziehe ich meinen Hund?, das im ersten Herr Bello-Band genannt wird, wäre darüber hinaus das Verfassen eines Kapitels aus dem Ratgeber Wie erziehe ich meinen Menschen? denkbar. Kreative Schreibaufgaben wie die hier vorgeschlagenen

29 Vgl. hierzu die Problematisierung, die Andrea Klatt in ihrem Beitrag im vorliegenden Band vornimmt.

30 Vgl. P. Maar: Wiedersehen mit Herrn Bello, S. 8

31 Vgl. R. Heuberger: Sprachgebrauch, S. 51.

32 Vgl. Schröder, Klarissa: »Moralische Handlungsanforderungen am Beispiel der Haustierhaltung in >tierfreundlichen Kinderbüchern««, in: Dies./Björn Hayer (Hg.), Didaktik des Animalen. Vorschläge für einen tiergestützten Literaturunterricht, Trier: WVT 2016, S. 197-211. 
regen dazu an, sich von tradierten Denkmustern zu lösen und neue Weltentwürfe in eigenen Texten konsequent weiterzudenken und weiterzuentwickeln.

Schließlich können Mensch-Tier-Hierarchien durch szenische Verfahren transparent gemacht werden. Das ist natürlich immer durch Aufgabenformate möglich, in denen die Schülerinnen und Schüler die Rolle eines Tieres einnehmen und aus dessen Perspektive sprechen und argumentieren, etwa in Rolleninterviews, wie Ingo Scheller sie anregt. ${ }^{33}$ Die Behandlung Bellos im Zirkus birgt darüber hinaus die Gelegenheit, sich mit verschiedenen Sichtweisen auf den Tierschutz zu beschäftigen und daraus eine Gerichtsverhandlung zu entwickeln. Vater Sternheim und Sohn Max könnten in diesem Fall als Kläger auftreten, die den Zirkus beschuldigen, Bellos Rechte im Sinne des Tierschutzgesetzes $\S 1$ und $\S 2$, die ausdrücklich auch für Zirkusbetriebe gelten, verletzt zu haben. Dadurch dass die verschiedenen menschlichen Positionen - Hundebesitzer, Zirkus, Zirkusbesucher -, aber auch das Tier selbst zu Wort kommen, werden die juristische Komplexität des Themas sowie die unterschiedlichen Interessen und Perspektiven deutlich.

\section{DIDAKTISCHE DIMENSIONEN}

Perspektivität ist eine zentrale Kategorie sowohl der Tierethik als auch des literarischen Lernens. »[D]ie Perspektive und die Situation anderer aus ihrer Sicht zu verstehen $\ll,{ }^{34}$ ist beispielsweise für Lori Gruen ein tierethischer Grundsatz, wenn man, so der Titel ihres Aufsatzes, [s]ich Tieren zuwenden will. Und »Perspektiven literarischer Figuren nachvollziehen" ist nach Kaspar H. Spinner ein bedeutsamer Aspekt im Bereich des literarischen Lernens. ${ }^{35}$ Im Rahmen seiner Studien zur Perspektivübernahme weist Florian Rietz zusätzlich auf die besonderen Herausforderungen des ersten Herr Bello-Romans hin und betont, dass der Leser auf der dritten und höchsten Stufe der Perspektivübernahmekompetenz, die er als Perspektivrelativierung bezeichnet,

33 Vgl. Scheller, Ingo: Szenische Interpretation. Theorie und Praxis eines handlungs- und erfahrungsbezogenen Literaturunterrichts in Sekundarstufe I und II, Seelze: Kallmeyer ${ }^{2} 2008$, S. 68 f.

34 Gruen, Lori: »Sich Tieren zuwenden: Empathischer Umgang mit der mehr als menschlichen Welt«, in: Friederike Schmitz (Hg.), Tierethik. Grundlagentexte, Berlin: Suhrkamp 2014, S. 390-404, hier S. 404.

35 Spinner, Kaspar H.: »Literarisches Lernen«, in: Praxis Deutsch (2006) 200, S. 6-16, hier S. 9f. 
»in der Lage [sei], seine Perspektive zu relativeren und seine mentalen Konzepte zu überdenken bzw. seinem Wissen anzupassen. Er erkennt, dass es andere Werte- und Normensysteme gibt. So steht bei den angeführten Beispielen die Frage im Mittelpunkt, ob die Leser das Verhalten von Herrn Bello als falsch einzuschätzen haben oder ob dessen Handeln aus seiner Hund-Perspektive richtig ist, da er als Hund ein anderes Werte- und Normensystem kennt. [...] Für den Leser gilt es, die eigene Perspektive dahingehend zu relativieren, dass Herr Bello trotz seiner Erscheinung kein Mensch ist und sein Verhalten auch nicht unter menschlichen Aspekten beurteilt werden darf. « ${ }^{36}$

Mit dem Aspekt der Perspektivität hängt jener der Empathie eng zusammen. »Empathie ist«, so konstatiert Michael Rosenberger aus moraltheologischer Sicht, »weit mehr als nur ein Gefühl«:

»Als ethische Grundhaltung will sie ständig mehr gelernt, geübt und weiterentwickelt werden. Erkenntnistheoretisch verkörpert sie einen Perspektivenwechsel [...]. Die Frage >wie würde es mir gehen, wenn ich in der Situation des anderen Geschöpfs wäre?« führt dann über Analogieschlüsse zu differenzierten Handlungsperspektiven und zum qualifizierten Handeln. $\ll^{37}$

Es geht also im tierethischen Kontext weniger um den rationalen Akt der Perspektivübernahme als vielmehr um »Emotionseinnahme«, die Ralph Olsen im Empathie-Diskurs als »eine stärker emotional orientierte aktive Tätigkeit « ${ }^{38}$ definiert.

Figuren, die sowohl die menschliche als auch die tierliche Sicht auf die Welt kennen und erlebt haben, sind, wie eingangs bemerkt, ein Privileg der Literatur, weshalb sich Tierethik und Literaturdidaktik hier ideal verbinden lassen. Wenn Tiere, wie in Paul Maars Herr Bello-Trilogie, zu literarischen Figuren werden, wenn sie sprechen, wenn sie als Erzählinstanzen im narratologischen Sinn fungieren, wenn sie ihre Sichtweise der menschlichen entgegenhalten oder die tierliche und die menschliche Perspektive verschwimmen, kann eine Didaktik des Animalen hier in besonderem Maße ansetzen und, wie es Gabriela Kompatscher als ein zentrales

36 Rietz, Florian: ")Was ist denn Herr Bello?〈 Aspekte einer Perspektivübernahmekompetenz am Beispiel von Paul Maars Herr Bello und das blaue Wunder«, in: Wicke/Roßbach, Paul Maar (2017), S. 273-288, hier S. 287.

37 Rosenberger, Michael: »Mit Noah in der Arche - mit Jesus im Paradies. Neuere Ansätze der theologischen Tierethik«, in: Herwig Grimm/Carola Otterstedt (Hg.), Das Tier an sich. Disziplinübergreifende Perspektiven für neue Wege im wissenschaftsbasierten Tierschutz, Göttingen: Vandenhoeck \& Ruprecht 2012, S. 14-36, hier S. 20.

38 Olsen, Ralph: »Das Phänomen Empathie beim Lesen literarischer Texte. Eine didaktischkompetenzorientierte Annäherung«, in: zeitschrift ästhetische bildung 3 (2011) 1, S. 1-16, hier S. 11. 
Ziel der Human-Animal Studies formuliert, »zu einer geänderten Wahrnehmung von Tieren führen «. ${ }^{39}$

Dabei muss allerdings mit den Schülerinnen und Schülern auch geklärt werden, dass wir trotz empathischer Annäherung »nicht wissen, wie es ist, ein Tier zu sein, da wir uns lediglich vorstellen können, wie es für uns Menschen wäre, sich wie das jeweilige Tier zu verhalten «. ${ }^{40}$

Im »Umgang mit Literatur«, so postuliert es beispielsweise der Hessische Lehrplan für das Fach Deutsch, soll die Bereitschaft der Schülerinnen und Schüler »gefördert werden, ihre Beziehung zu anderen Menschen nach den Grundsätzen der Toleranz, Gerechtigkeit und Solidarität zu gestalten. « ${ }^{41}$ Dass dies durchaus auch auf den Umgang mit Tieren übertragbar ist, haben die hier angestellten Überlegungen zu zeigen versucht. Das allerdings wird im Lehrplan noch nicht berücksichtigt.

39 Kompatscher, Gabriela: »Literaturwissenschaft. Die Befreiung ästhetischer Tiere«, in: Reingard Spannring/Karin Schachinger et al. (Hg.), Disziplinierte Tiere? Perspektiven der Human-Animal Studies für die wissenschaftlichen Disziplinen, Bielefeld: transcript 2015, S. 137-159, hier S. 154.

40 Vgl. Kompatscher/Spannring et al.: Human-Animal Studies (2017), S. 38.

41 https://kultusministerium.hessen.de/sites/default/files/media/g8-deutsch.pdf, letzter Abruf am 20.07.2018. 